\title{
Recombinant adenovirus expressing a dendritic cell-targeted melanoma surface antigen for tumor-specific immunotherapy in melanoma mice model
}

\author{
LI-LI GUO, GANG-CHENG WANG，PENG-JIE LI，CUI-MEI WANG and LIN-BO LIU
}

\author{
Department of Plastic Surgery, The First Affiliated Hospital, Zhengzhou University, Zhengzhou, Henan 450052, P.R. China
}

Received September 27, 2016; Accepted June 5, 2017

DOI: $10.3892 /$ etm.2018.6085

\begin{abstract}
Viral vectors represent a potential strategy for the treatment of human malignant tumors. Currently, recombinant adenovirus vectors are commonly used as gene therapy vehicles, as it possesses a proven safety profile in normal human cells. The recombinant adenovirus system has an ability to highly express exogenous genes and increase the stability of the carrier, which is only transiently expressed in the host cell genome, without integrating. Malignant melanoma cells are produced by the skin, and melanocyte tumors that exhibit higher malignant degrees lead to earlier transfer and higher mortality. In the present study, a recombinant adenovirus (rAd) was generated to express Anti-programmed death-1 (rAd-Anti-PD-1) and used to investigate the efficacy in melanoma cells and tumors. The results demonstrated that B16-F10 cell growth was significantly inhibited and the apoptosis incidence rate was markedly promoted following rAd-PD-1 treatment. The present study demonstrated that the production of $\alpha$ and $\beta$ interferon was increased, which led to the induction of dendritic cell (DCs) maturation in rAd-anti-PD-1-treated mice. The present study indicated that rAd-anti-PD-1 exhibited the ability to generate more cluster of differentiation (CD) $4^{+} \mathrm{CD}^{+} \mathrm{T}$ cells and induce a PD-1-specific cytotoxic $\mathrm{T}$ lymphocyte through DC-targeted surface antigens in mice. This resulted in a further enhanced recognition of melanoma cells due to DCs being targeted by the rAd-anti-PD-1-encoded PD-1. Notably, mice treated with the rAd-anti-PD-1-targeted PD-1 demonstrated an improved protection compared with tumor-bearing mice from the challenge group treated with a recombinant gutless adenovirus and Anti-PD-1. In conclusion, the present study demonstrated that targeting the melanoma surface antigens via the rAd-anti-PD-1-infected tumor cells
\end{abstract}

Correspondence to: Professor Lin-Bo Liu, Department of Plastic Surgery, The First Affiliated Hospital, Zhengzhou University, 100 Kexueda Raod, Zhengzhou, Henan 450052, P.R. China E-mail: liulinboprof@163.com

Key words: melanoma, recombinant adenovirus expressinganti-programmed death-1, anti-programmed death-1, dendritic cells, cytotoxic T lymphocyte enhanced the ability of recombinant adenovirus to induce a potent tumor-inhibitory effect and antigen-specific immune response.

\section{Introduction}

Melanoma derived from melanocytes that exhibit the capacity to be more aggressive and a marked resistance to treatment is leading to an increased morbidity (5\%) and mortality rates (15\%) globally (1). Advanced melanoma has historically been associated with a poor prognosis and a 5-year survival rate of $<10 \%(2,3)$. The most important clinical features of melanoma in the initial stages (I/II) are the local migration and long distance metastases through the lymph nodes $(4,5)$. In addition, previous studies have demonstrated that melanoma frequently metastasized to other organs, which is another factor that contributes to the low survival rate $(6,7)$. Although surgery, conventional and comprehensive treatments may produce modest benefits to patients and improve patients' survival; clinical trials have indicated that the short survival time of patients with melanoma remained and $<15 \%$ of patients survived $(8,9)$. Therefore, investigating a more efficient approach to inhibit the local and long distance migration of melanoma metastases may improve the survival rate of patients.

Programmed death-1 (PD-1) is a type of immunosuppressive molecule that is activated by programmed death receptor $1(10,11)$. PD-1 is a member of the cluster of differentiation 28 (CD28) superfamily, which originated and was cloned through the apoptosis of T cells in mice hybridoma (12). Targeting PD-1 immunity against tumors, anti-infection, autoimmune disease and organ transplantation survival have demonstrated marked outcomes in previous studies $(13,14)$. PD-1 ligand L1 and the antibody of PD-1 are able to produce the same effects via binding with PD-1 (15). Notably, a previous study has indicated that PD-1 is expressed on the surface of various malignancies, including hepatoma, non-small cancer lung cell and colon cancer (16). The antibody target for PD-1 (anti-PD-1) was able to inhibit PD-1 expression (17). However, the function of anti-PD-1 and the survival of patients with cancer remain to be elucidated. Furthermore, the role of PD-1 expression on melanoma cells remains unknown. The present study investigated the therapeutic effects of anti-PD-1 in a mouse model of melanoma. Tumor-bearing mice were used to evaluate the efficacy of anti-PD-1 and the overall survival 
according to PD-L1 status in mice with melanoma was assessed by meta-analysis.

Previous studies have indicated that a recombinant adenovirus constructed based on the Adeno-X expression system, resulted in a gene therapy vehicle to treat human cancer $(18,19)$. Adenovirus vectors are one of the most widely used vectors in gene therapy applications for the treatment of diverse human diseases, including cancer (20). The immunotherapy accompanied with other therapeutic methods has revealed therapeutic effects in animal models and different clinical trials (21-23). The use of antineoplastic drugs combined with immunotherapy has been demonstrated to effectively target tumor cells with specific recognition molecules or domains of antigens or receptors (24-27). A number of different agents for the treatment of cancer, including chemoradiotherapy and immunotherapy, are being assessed in preclinical trials $(28,29)$. Immunotherapy agents, in which an antibody or interleukin is inserted into a gene expression vector, have led to positive outcomes regarding tumor apoptosis in patients with advanced tumors pre-clinically and clinically (30). The most crucial area of gene therapy is to establish an effective gene delivery system. In the present study, the oncolytic adenovirus-mediated gene therapy system expressing anti-PD-1 was investigated as a treatment for melanoma-bearing mice.

Development of novel and effective clinical protocols for cancer therapy is urgently required in modern medicine (31). Previously, immunotherapies against the growth and metastasis of tumor cells have been revealed to be a potentially promising approach for the treatment of human cancer (32). The antitumor efficacy of the $\mathrm{T}$ cell immune response depends on the exposure of tumor antigens and activities of antigen-presenting cells, which may efficiently present tumor antigens leading to the activation of cytotoxic $\mathrm{T}$ lymphocytes. Notably, oncolytic adenovirus expressing interferon- $\gamma$ $(\mathrm{IFN}-\gamma)$ resulted in significant tumor growth suppression in a syngeneic Syrian hamster model for the treatment of pancreatic cancer (33). This in turn, eradicates corresponding tumor cells. In addition, immunotherapies are activated by dendritic cells (DCs) loaded with tumor surface antigens including tumor cell lysates, nucleic acids encoding tumor antigens, tumor-specific proteins, apoptotic bodies and necrotic tumor cells. A number of research papers have demonstrated a strategy for producing mature DCs, influenced by tumor phenotype and immune responses for eradicating tumor cells. Furthermore, targeting the tumor specific antigens by treating the antibody specific to the DC-restricted antigen may elevate exposure of the tumor specific antigen. This may promote recognition of tumor specific antigens resulting in a limitation in tumor capacity $(34,35)$. The present study demonstrated that the targeting of the melanoma antigen to DC cells via anti-PD-1 expressed by recombinant adenovirus expressing (rAd)-PD-1 markedly promoted the ability of rAd to induce responses from melanoma antigen-specific cytotoxic T lymphocytes.

\section{Materials and methods}

Cells and reagents. Melanoma cell lines, B16-F10 and SkMel-2, and a normal human epidermal cell line (NHEC; NHEK) were purchased from the American Type Culture Collection (ATCC; Manassas, VA, USA) and cultured in minimum essential media (MEM) supplemented with $10 \%$ fetal bovine serum (both from Gibco; Thermo Fisher Scientific, Inc., Waltham, MA, USA) and $2 \mathrm{mM}$ penicillin/streptomycin (Sigma-Aldrich; Merck KGaA, Darmstadt, Germany) in a humidified incubator at $37^{\circ} \mathrm{C}$ and $5 \% \mathrm{CO}_{2}$.

Construction of recombinant adenovirus. An adeno-X expression system (A4470; Sigma-Aldrich; Merck KGaA) (36) was used to construct the recombinant adenovirus constructs. DNA sequence (GenBank, NM_008798.2) encoding full-length Anti-PD-1 linked with the cell-penetrating peptide and Fc was cloned into the rAd-X plasmids (A4470; Sigma-Aldrich; Merck KGaA) by polymerase chain reaction (PCR) using KAPA Taq PCR kit with dNTPs (cat. no. TAQNTKB; Sigma-Aldrich; Merck KGaA). The primers were as follows: PD-1 forward, 5'-TCGATCTGGAACTGTGGCCAT-3' and reverse, 5'-TGGC CAGGGCGCCTGTGGATCTAA-3'. Then, the recombinant adenoviral plasmid rAd-anti-PD-1 and rAd-enhanced green fluorescent protein (rAd-EGFP, produced in our laboratory) were produced by PCR as described previously for subsequent analysis. PCR and sequencing (Invitrogen; Thermo Fisher Scientific, Inc.) were used to identify recombinant adenovirus constructs. Thermocycling of PCR was performed using PCR kit (11696505001; Sigma-Aldrich; Merck KGaA) as follows: $95^{\circ} \mathrm{C}$ for $1 \mathrm{~min}$; and 25 cycles of $94^{\circ} \mathrm{C}$ for $30 \mathrm{sec}, 56^{\circ} \mathrm{C}$ for $1 \mathrm{~min}$ and $72^{\circ} \mathrm{C}$ for $1 \mathrm{~min}$. The recombinant adenoviruses were generated by transfecting into HEK293 cells using Lipofectamine 2000 (Sigma-Aldrich; Merck KGaA) according to manufacturers' instructions and underwent culture in DMEM at $37^{\circ} \mathrm{C}$ in an atmosphere containing $5 \% \mathrm{CO}_{2}$. The recombinant adenovirus constructs were purified as described in a previous study (18). Adenovirus titers were determined by TCID $_{50}$ as plaque-forming units/ml using the Reed-Muench method (37).

MTT assay. B16-F10 and SkMel-2 cells $\left(1 \times 10^{3}\right)$ were cultured and then inoculated with rAd-Anti-PD-1 [0.5 multiplicity of infection (MOI)] or rAd-EGFP 0.5 (MOI) or PBS in 96-well plates for $48 \mathrm{~h}$ at $37^{\circ} \mathrm{C}$ in triplicate for each condition. Following culture, $20 \mu \mathrm{l}$ MTT $(5 \mathrm{mg} / \mathrm{ml})$ in PBS solution was added to each well, the plate was further incubated for $4 \mathrm{~h}$ at $37^{\circ} \mathrm{C}$. The medium was entirely removed and $100 \mu \mathrm{l}$ dimethyl sulfoxide was added to the wells to solubilize the crystals. The optical density (OD) was measured using an ELISA microplate reader (Bio-Rad Laboratories, Inc., Hercules, CA, USA) reader at a wavelength of $450 \mathrm{~nm}$.

Cells morphology. B16-F10 and SkMel-2 cells $\left(5 \times 10^{6}\right)$ were cultured in six-well plate and treated with rAd-Anti-PD-1 (0.5 MOI), rAd-EGFP, rAd or vehicle (Mock) in 6-well plates for $48 \mathrm{~h}$ at $37^{\circ} \mathrm{C}$. The cells were cells morphology was observed by a microscope (Nikon E400, Nikon Instrument Group, Japan). The EGFP fluorescence was observed using a confocal microscope (magnification, x40; Olympus FV10; Olympus, Tokyo, Japan).

Reverse transcription-quantitative PCR (RT-qPCR). Total RNA was extracted from B16-F10 and SkMel-2 cells using 
the RNAeasy Mini kit (Qiagen GmbH, Hilden, Germany). The mRNA expression of PD-1 in B16-F10 and SkMel-2 cells was measured using an RT-qPCR kit (Roche Diagnostics, Mannheim, Germany) with $\beta$-actin expression as an endogenous control (Qiagen $\mathrm{GmbH}$ ) according to the manufacturer's instructions. All primers were synthesized by Invitrogen (Thermo Fisher Scientific, Inc., Waltham, MA, USA). The following primers were used: PD-1 forward, 5'-AAGTTTCAGGGAAGGTCAG-3' and revers, 5'-CTGGGC ATGTGTAAAGGT-3'; and $\beta$-actin forward, 5'-CTAAGTCAT AGTCCGCCTAGAAGCA-3' and reverse, 5'-CTAAGTCAT AGTCCGCCTAGAAGCA-3'. PCR reactions contained $25 \mathrm{ng}$ cDNA template, $100 \mathrm{ng}$ forward and reverse oligonucleotide primers, $2.5 \mu 1$ Taq PCR buffer (Promega Corporation, Madison, WI, USA), $0.4 \mathrm{mM}$ dNTP mixture and $1 \mathrm{U}$ Taq polymerase (Promega Corporation, Madison, WI, USA) in a total reaction volume of $25 \mu \mathrm{l}$. After $120 \mathrm{sec}$ incubation at $95^{\circ} \mathrm{C}$, PCR was performed using 25 cycles of denaturation at $94^{\circ} \mathrm{C}$ for $30 \mathrm{sec}$, annealing at $54^{\circ} \mathrm{C}$ for $30 \mathrm{sec}$ and elongation at $72^{\circ} \mathrm{C}$ for $30 \mathrm{sec}$. Relative PD-1 expression level was calculated using the $2^{-\Delta \Delta \mathrm{Cq}}$ method (38). The results were presented as the $n$-fold change compared with $\beta$-actin using Quantiscan2.1 (Software Demo of AB QuantStudio ${ }^{\text {TM }}$ 12K Flex System; Thermo Fisher Scientific, Inc.).

Cells invasion and migration assays. B16-F10 and SkMel-2 cells were cultured in DMEM medium at $37^{\circ} \mathrm{C}$ in an atmosphere containing $5 \% \mathrm{CO}_{2}$ and treated with rAd-Aiti-PD-1 (0.5 MOI), rAd-EGFP (0.5 MOI) or PBS as a control. For the invasion assay, virus-treated cells were suspended at a density of $1 \times 10^{6}$ cells $/ \mathrm{ml}$ in serum-free MEM and then transferred to the upper chamber of a BD BioCoat Matrigel Invasion Chamber (BD Biosciences, San Jose, CA, USA) according to the manufacturer's instructions. The cells were fixed using methanol for $30 \mathrm{~min}$ at $37^{\circ} \mathrm{C}$ and stained for $30 \mathrm{~min}$ in a $0.1 \%$ Crystal Violet solution in $\mathrm{PBS}$ at $37^{\circ} \mathrm{C}$. Invasion and migration were calculated in at least three random fields of view under a microscope (Nikon E400; magnification, x20; Nikon Instrument Group, Tokyo, Japan).

Animal study. A total of 80 eight-week-old male specific-pathogen-free C57BL/6 nude immunodeficiency mice (30-35 g) were purchased from Shanghai Laboratory Animal Centre (Shanghai, China). All animals were housed in a temperature-controlled facility at $23 \pm 1^{\circ} \mathrm{C}$ (humidity, $50 \pm 5 \%$ ) with a 12-h light/dark cycle. All rats had free access to food and water. A total volume of $100 \mu 1 \mathrm{~B} 16-\mathrm{F} 10$ cells $\left(1 \times 10^{7}\right)$ were administered subcutaneously into a site on the back of the C57BL/6 mice. C57BL/6-bearing mice were randomly divided into four groups and received treatment of rAd-Aiti-PD-1 (0.5 MOI), rAd-EGFP (0.5 MOI), rAd (0.5 MOI) or PBS $(n=20$ in each group). The therapy (rAd, rAd-EGFP or rAd-anti-PD-1, $5 \mathrm{MOI}$ ) was initiated on day 6 when tumor diameters reached 5-6 $\mathrm{mm}$ and mice were treated 10 times for a period of 20 days (i.e., every other day). Mice were sacrificed when tumor diameters reached $12 \mathrm{~mm}$. Tumor diameters were recorded every 2 days and tumor volume was calculated as follows: $0.52 \times$ smallest diameter ${ }^{2}$ x largest diameter (39). The present study was performed according to the recommendations in the Guide for the Care and Use of Laboratory Animals (40).
All experiments were completed in accordance with National Institutes of Health and approved by Committee on the Ethics Committee of Zhengzhou University (Zhengzhou, China). All surgeries and euthanasia were performed under sodium pentobarbital anesthesia $(40 \mathrm{mg} / \mathrm{kg})$ when tumor diameter reached $16 \mathrm{~mm}$.

Splenocyte collection and cytotoxic T lymphocyte (CTL) responses. Splenocytes were extracted from spleens of the experimental animals using cell separation method described previously (41) ( $\mathrm{n}=4$ in each group) on day 30 after tumor incubation. The monoplast suspension was washed three times with PBS three times at $37^{\circ} \mathrm{C}$. Then, inactivated B16-F10 cells $\left(1 \times 10^{6}\right)$ were incubated with splenocytes $\left(1 \times 10^{4}\right)$ for $12 \mathrm{~h}$ at $37^{\circ} \mathrm{C}$. Release of interferon (IFN) was evaluated by ELISA (DY485, Bio-Rad Laboratories, Inc., Hercules, CA, USA) in the supernatants following culture for $72 \mathrm{~h}$. T cells $\left(1 \times 10^{6}\right)$ from the splenocytes were purified, as previously described (32) and co-cultured with fresh DMEM B16-F10 cells for $4 \mathrm{~h}$ at $37^{\circ} \mathrm{C}$ at the effector, at target ratios of 5:1, 20:1 and 40:1, which were ratios used in a previous study (42). Specific CTL activity to the target cells was determined by MTT cytotoxicity assays, as previously described (43).

Western blot analysis. B16-F10 and SkMel-2 cells $\left(1 \times 10^{6}\right)$ were cultured, lysed and used to analyze PD-1 expression according to a previous study (44). Cells were homogenized in lysate buffer containing protease-inhibitor (Sigma-Aldrich; Merck KGaA) and were centrifuged at $8,000 \times \mathrm{g}$ at $4^{\circ} \mathrm{C}$ for $10 \mathrm{~min}$. Protein concentration was measured using the BCA protein assay kit (Thermo Scientific Fisher Scientific, Inc.). Protein samples (20 $\mu \mathrm{g}$ per lane) were separated using $15 \%$ SDS-PAGE and transferred onto polyvinylidene difluoride membranes (EMD Millipore, Billerica, MA, USA). Following this, blocking with $5 \%$ bovine serum albumin was performed at $37^{\circ} \mathrm{C}$ for $1 \mathrm{~h}$. The following primary antibodies were used in immunoblotting assays: PD-1 (1:1,000, cat. no. ab63477) and $\beta$-actin $(1: 1,000$, cat. no. ab8226; all from Abcam, Cambridge, UK) for $12 \mathrm{~h}$ at $4^{\circ} \mathrm{C}$. Horseradish peroxidase-conjugated antibody (1:5,000; cat. no: HAF019, Bio-Rad Laboratories, Inc.) was used as a secondary antibody for $2 \mathrm{~h}$ at $37^{\circ} \mathrm{C}$. Subsequently, bands were detected using a western blotting Luminol reagent (cat. no. 12015218001; Sigma-Aldrich; Merck KGaA). The density of the bands was analyzed by Quantity one software version 4.62 (Bio-Rad Laboratories, Inc.).

Histological analysis. Tumor tissues from experimental mice were fixed using $10 \%$ formaldehyde for $2 \mathrm{~h}$ at $37^{\circ} \mathrm{C}$ followed by being embedded in paraffin. Tumor samples were cut into sections $(4 \mu \mathrm{m})$ and antigen retrieval (at $95^{\circ} \mathrm{C}$ for $15 \mathrm{~min}$ ) was also performed on tumor sections. Tumor sections were incubated with primary antibodies: PD-1 (1:1,000, ab214421, Abcam, Cambridge, MA, USA) and $\beta$-actin (1:1,000, ab8226, Abcam). Subsequently, sections were incubated with horseradish peroxidase (HRP)-conjugated polyclonal anti-rabbit immunoglobulin $\mathrm{G}$ antibody (1:10,000; R\&D Systems, Inc., Minneapolis, MN, USA) for $1 \mathrm{~h}$ at room temperature. A Ventana Benchmark automated staining system (Ventana Medical Systems, Inc.; Roche Holding AG, Basel, Switzerland) 
A

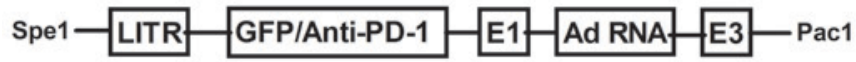

B

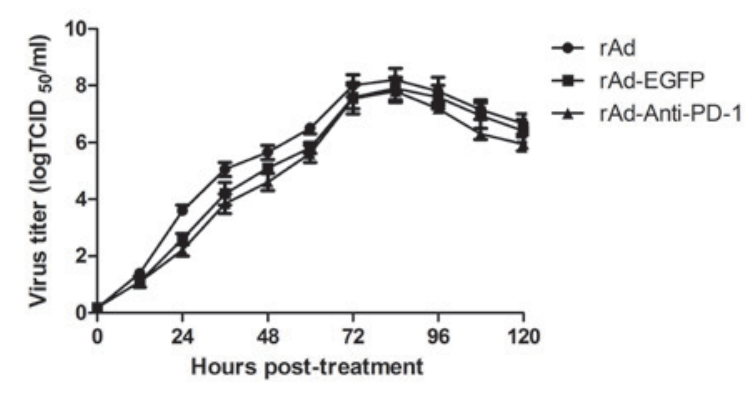

D
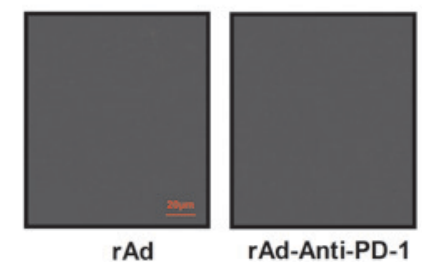

rAd-Anti-PD-1

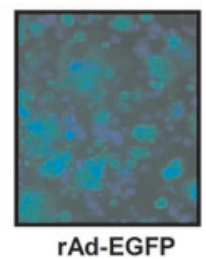

Figure 1. Characterization of the recombinant adenovirus. (A) Schematic diagram of rAd-EGFP and rAd-anti-PD-1. (B) Virus titer of rAd-EGFP and rAd-anti-PD-1 with rAd as a control. (C) Concentration level of anti-PD-1 analysis in melanoma cells following infection with rAd-anti-PD-1. (D) EGFP syncytia formation was observed in anti-EGFP-infected cells viewed by fluorescent microscopy (magnification, $\mathrm{x} 40$ ). ${ }^{* *} \mathrm{P}<0.01 \mathrm{vs}$. control. Data are presented as mean \pm standard error of the mean. Ad, adenovirus; rAd, recombinant adenovirus; EGFP, enhanced green fluorescence protein; PD-1, programmed death-1; LITR, left inverted terminal repeat; TCID, tissue culture infective dose.

was used for observing the protein expression (Olympus BX51, Olympus; Tokyo, Japan; magnification, x20).

Statistical analysis. All data were reported as means \pm standard error of the mean from triplicate experiments. Data was analyzed using GraphPad Prism 6.0 software (GraphPad Software, Inc., La Jolla, CA, USA). Unpaired data was analyzed by Student's t-test. Comparisons of data between multiple groups were analyzed by one-way analysis of variance followed by Tukey HSD test. $\mathrm{P}<0.05$ was considered to indicate statistically significant differences.

\section{Results}

Characterization of the recombinant adenovirus constructs. The recombinant adenovirus constructs delivering the Anti-PD-1 or EGFP were generated based on the adeno-X expression system. The description of adenovirus constructs is presented in Fig. 1A. In order to investigate whether the insertion of the foreign gene in the adeno-X expression system affected the growth of the virus, the kinetic growth of recombinant adenovirus constructs was analyzed. The result in Fig. 1B demonstrated that similar virus titers were observed; indicating that insertion of the foreign gene in the adeno-X expression system did not significantly affect replication and growth kinetics of recombinant adenovirus constructs. In addition, the expression level of foreign gene expression levels was analyzed in the virus-infected B16-F10 and SkMel-2 cells. The results indicate that the Anti-PD-1 vector was efficiently expressed and secreted into extracellular space $72 \mathrm{~h}$ after infection (Fig. 1C). Furthermore, EGFP syncytia formation by Anti-EGFP-infected cells was observed by fluorescent microscopy (Fig. 1D). Collectively, the data suggests that insertion of the foreign gene in the adeno-X expression system do not affect viral growth and expression in tumor cells.

rAd expressing Anti-PD-1 targeted PD-1 and effectively enhanced the lysis of human and murine melanoma cell lines. The PD-1 expression in B16-F10 and SkMel-2 cells was further assessed using RT-qPCR. The results indicated that PD-1 expression was higher in B16-F10 and SkMel-2 cells compared with NHECs ( $\mathrm{P}<0.01$; Fig. 2A). Notably, rAd-anti-PD-1 significantly decreased the expression of PD-1 at $48 \mathrm{~h}$ following inoculation $(\mathrm{P}<0.05$; Fig. $2 \mathrm{~B})$. To further confirm that the anti-PD-1 protein exhibited a higher expression in tumor cells infected by rAd-anti-PD-1, the present study performed a time course of rAd-anti-PD-1 infection in B16-F10 cells. The results in Fig. 2C indicate that anti-PD-1 was detected as early as $6 \mathrm{~h}$ following infection with rAd-anti-PD-1 at $0.1 \mathrm{MOI}$. Furthermore, rAd-anti-PD-1 effectively lysed melanoma cell lines compared with rAd-EGFP and rAd (Fig. 2D). Taken together, these results suggest that rAd-anti-PD-1 is efficiently expressed in tumor cells and was able to downregulate PD-1 expression.

rAd expressing Anti-PD-1 enhanced antitumor efficacy in B16-F10-xenograft mice. In order to detect the inhibitory effects of rAd-Anti-PD-1, tumor cell growth was analyzed in vitro. The growth of B16-F10 cells was significantly inhibited following $\mathrm{rAd}-\mathrm{Anti}-\mathrm{PD}-1$ treatment at $0.5 \mathrm{MOI}$ for $48 \mathrm{~h}$ compared with $\mathrm{rAd}$ and $\mathrm{rAd-EGFP}(\mathrm{P}<0.01$; Fig. $3 \mathrm{~A})$. In 
A

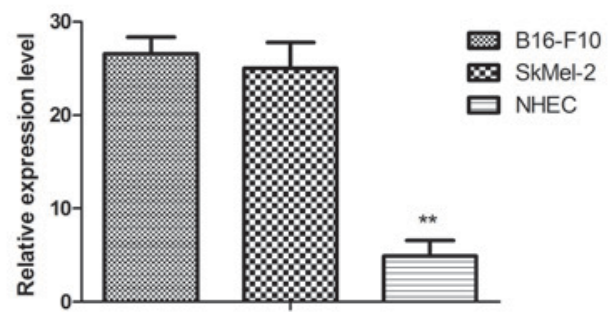

C

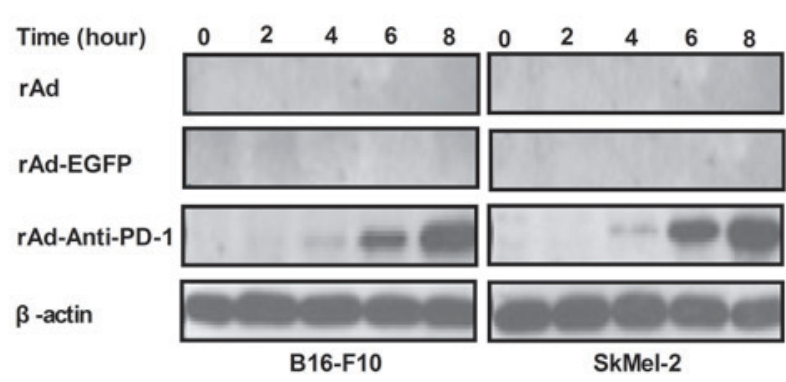

B

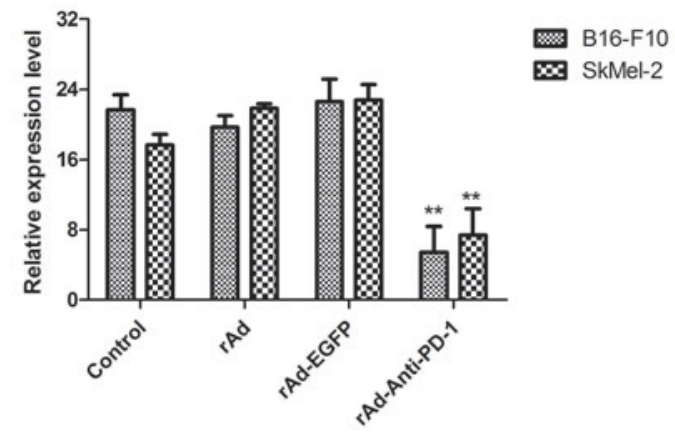

D

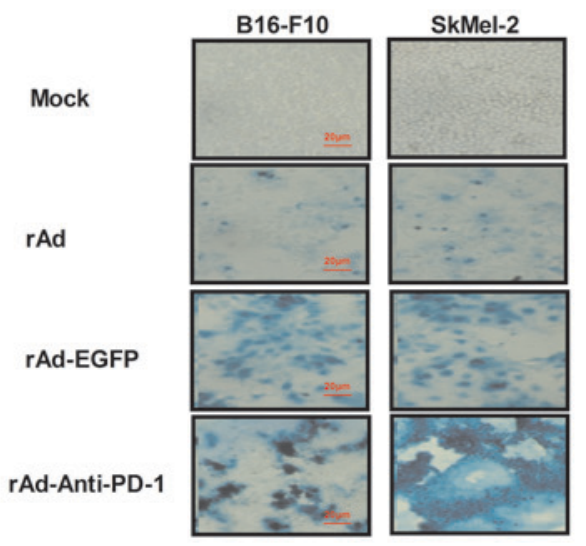

Figure 2. rAd-anti-PD-1 downregulated PD-1 expression and enhanced melanoma cell lysis. (A) Relative PD-1 mRNA expression in NHEC, B16-F10 and SkMel-2 cells. (B) PD-1 mRNA expression level was significantly decreased in B16-F10 and SkMel-2 cells following treatment with rAd-anti-PD-1. (C) Time course of anti-PD-1 protein expression levels in rAd-anti-PD-1-infected melanoma cell lines. (D) Efficiency of syncytia formation by treatment with rAd-anti-PD-1, observed by fluorescence microscopy (magnification, $\mathrm{x} 40$ ). Data are presented as mean \pm standard error of the mean. ${ }^{* *} \mathrm{P}<0.01$ vs. control. Mock, no virus-treated group; rAd, recombinant adenovirus expressing; PD-1, programmed death-1; NHEC, normal human epidermis cells.

addition, invasion of B16-F10 and SkMel-2 cells was significantly suppressed when treated with rAd-Anti-PD-1 at 0.5 MOI for $48 \mathrm{~h}$ compared with rAd and rAd-EGFP $(\mathrm{P}<0.01$; Fig. 3B). Subsequently, the efficacy of antitumor treatment with rAd-Anti-PD-1 was investigated in B16-F10-bearing mice. Animals were administered treatment of $\mathrm{rAd}$, rAd-EGFP, rAd-PD-1 or PBS as control by intravenous injection. These results demonstrate that rAd and rAd-EGFP treatment significantly inhibited tumor growth, compared with PBS as a control. Notably, a significant inhibition of tumor growth was observed with rAd and compared with rAd-anti-PD-1 ( $\mathrm{P}<0.01$; Fig. 3C). Furthermore, the 240-day long-term survival following treatment with rAd-anti-PD-1 was assessed and demonstrated that treatment with rAd-Anti-PD-1 ( $n=20)$ significantly prolonged the survival of mice compared with other groups $(\mathrm{P}<0.01$; Fig. 3D). Collectively, these results reveal that rAd-Anti-PD-1 significantly inhibited melanoma cell growth in vitro and in vivo, suggesting that the use of oncolytic therapy with rAd-anti-PD-1 against melanoma contributed to long-term survival of melanoma-bearing mice.

Induction of rAd-Anti-PD-1-specific cellular immune responses. A previous study indicated that presenting tumor antigen by DC is essential in order to activate cytotoxic $\mathrm{T}$ lymphocytes to inhibit the invasion of tumor cells (45).
Therefore, the present study analyzed the expression of B16-F10-sepcific tumor antigen and DC cells on the surface of tumor cells 21 days following tumor inoculation. The results indicated that the expression of tumor antigens and DC cells was elevated in rAd-anti-PD-1-treated tumors (Fig. 4A). The present study also revealed that PD-1 expression was markedly decreased, whereas apoptotic bodies were increased in tumors following rAd-anti-PD-1 treatment (Fig. 4B). In addition, the cytotoxic T lymphocyte response in rAd-Anti-PD-1-treated mice was also detected. As presented in Fig. $4 \mathrm{C}$, the production of IFN- $\alpha$ and IFN- $\beta$ increased, which contributed to induce the maturation of DCs in rAd-Anti-PD-1-treated mice. Furthermore, the results of the present study demonstrated that rAd-Anti-PD-1 exhibited the ability to generate significantly more $\mathrm{CD}^{+}$and $\mathrm{CD}^{+} \mathrm{T}$ cells and induce a $\mathrm{PD}-1$-specific CTL through DC-targeted surface antigens in mice that resulted in further enhancing recognition melanoma cells by the targeting of the rAd-anti-PD-1-encoded PD-1 to DCs $(\mathrm{P}<0.05$; Fig. 4D and $\mathrm{E})$. The present study revealed that melanoma-bearing mice treated with the rAd-Anti-PD-1-targeted PD-1 were significantly more protected against apoptosis than tumor-bearing mice following challenge with Anti-PD-1 (Fig. 4F). Collectively, the results of the present study confirmed the hypothesis and suggested that rAd-anti-PD-1 activated immunotherapy by inducing DC 
A

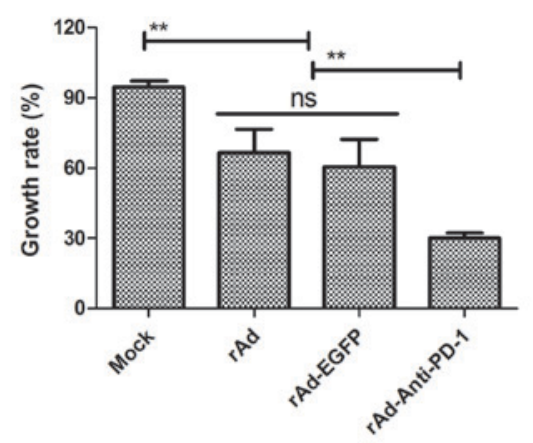

C

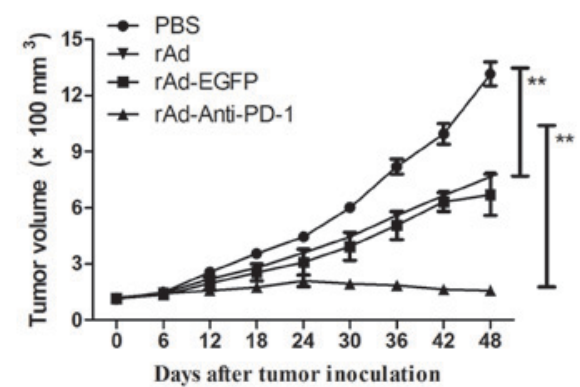

B

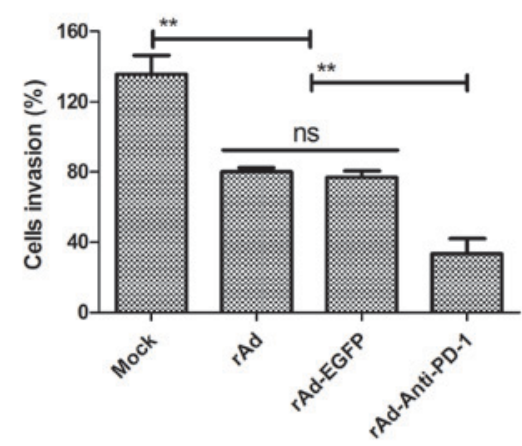

D

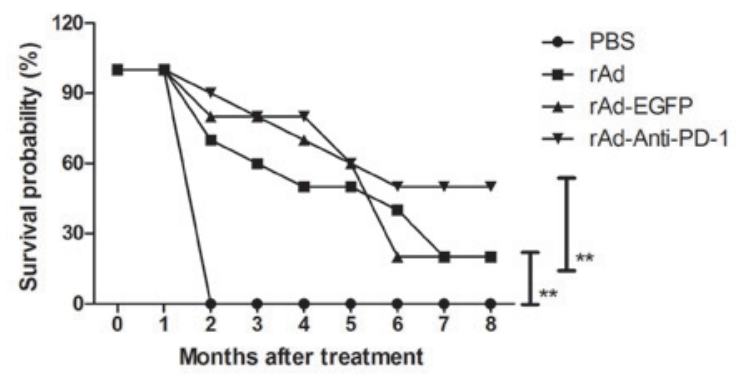

Figure 3. rAd-anti-PD-1 inhibited melanoma growth compared with rAd and rAd-EGFP in vitro and in vivo. (A) Growth of melanoma cells was suppressed following treatment with rAd-anti-PD-1 in vitro. (B) Invasion of melanoma cells was inhibited by rAd-anti-PD-1 treatment. (C) Melanoma tumor growth was inhibited following treatment with rAd-anti-PD-1 in vivo. (D) rAd-anti-PD-1 treatment prolonged survival of tumor-bearing mice in a 240-day observation. ${ }^{* *} \mathrm{P}<0.01$. Data are presented as mean \pm standard error of the mean. Mock, no virus-treated group; rAd, recombinant adenovirus expressing; PD-1, programmed death-1; EGFP, enhanced green fluorescence protein; PBS, phosphate-buffered saline.

maturation in melanoma, which contributed to tumor regression and long-term survival.

\section{Discussion}

Previous studies have indicated that gene therapy may provide an improved clinical method for treating melanoma, and these therapies were considered as potential adjuvants for other cancer therapies $(46,47)$. Adenovirus vectors are the most widely used treatment and Adenovirus-mediated delivery of functional genes or polypeptides into tumor cells is well understood (48). Additionally, gene transfer strategies have led to more clinicians using immunotherapy to treat patients with HCC, including inhibition of oncogenes and restoration of tumor-suppressor genes, immunotherapy, anti-angiogenesis and virotherapy (49). Therefore, the present study may provide a more effective therapy for melanoma tumors by delivering PD-1 via a recombinant adenovirus. The results of the present study indicated that PD-1 expressed by recombinant adenovirus induced the accumulation of DCs, which could expose tumor antigens and promote $\mathrm{T}$ lymphocyte-induced tumor cytotoxicity.

Gene therapy has previously identified potential candidates for the treatment of human diseases including cancer, cardiovascular disease, blood diseases, diabetes, genetic diseases and other diseases that cannot be treated with conventional drug therapies (50-52). Previously, the use of viral vectors to deliver vectors to express functional genes has been applied in different medical fields, including cancer therapy and cardiovascular disease. These oncolytic viral vectors have been demonstrated to be relatively safe due to a selectively to replicate in cancer cells, but not in normal cells $(53,54)$. The benefits of these oncolytic viral vectors are that as they replicate and lyse tumor cells, and functional genes delivered by oncolytic viral vectors strengthen the effects of tumor eradication and inhibit the chance of recurrence (55). Adenovirus, Newcastle disease virus and herpes simplex virus are the most commonly used gene therapy oncolytic viral vectors, as vectors that specifically mutate and selectively replicate faster in cancer cells (56). The Adeno-X expression system is the most commonly used and $>250$ patients have been treated with ONYX-015 (a replicating adenovirus) (57). In the present study, the Adeno-X expression system was used to deliver an antibody of PD-1 to assess the oncolytic effects of a recombinant virus in melanoma in vitro and in vivo.

CTL-associated PD-1 inhibitors are well understood and represent one of the most important immunomodulating agents (58). PD-1 receptor or antibody are well tolerated and exhibit a low rate of adverse effect recurrence, as demonstrated in a previous study (58). In addition, a study has demonstrated that antibodies targeting PD-1 signaling promote a T-cell-mediated antitumor therapy and prevent tumor invasion (59). The present study revealed that anti-PD-1 demonstrated a significant inhibition of melanoma cells growth and a marked increase of DC cell maturation to present melanoma-specific antigens for cytotoxic T lymphocyte-associated immunotherapy. A previous study indicated that DC cell maturation is an essential step for the development of anti-cancer 
A

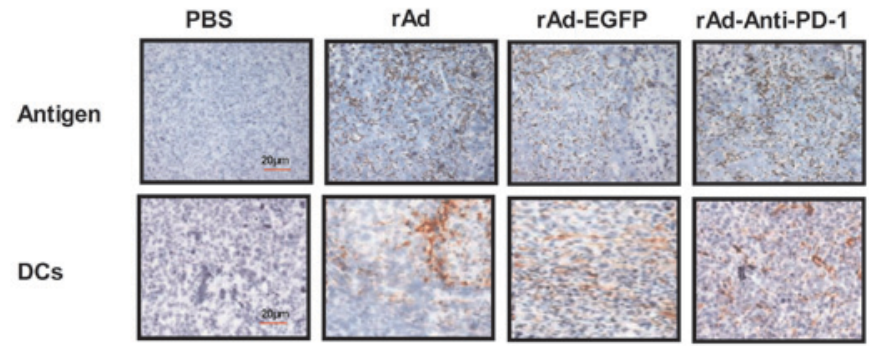

B

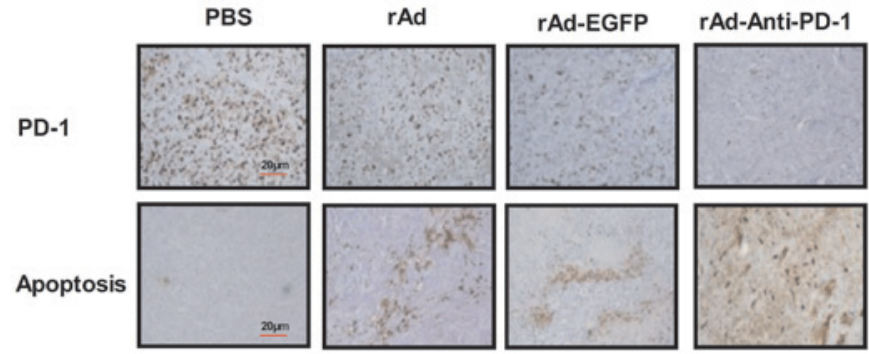

C

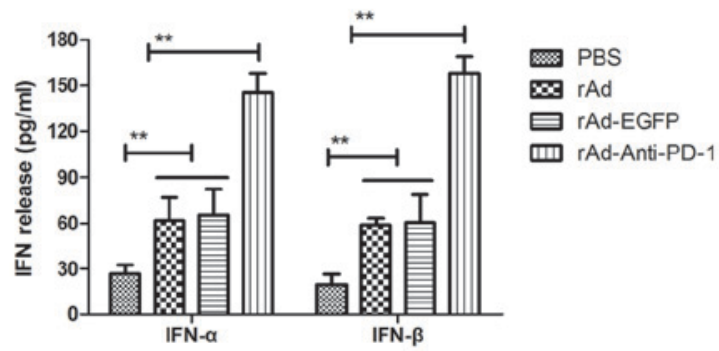

E

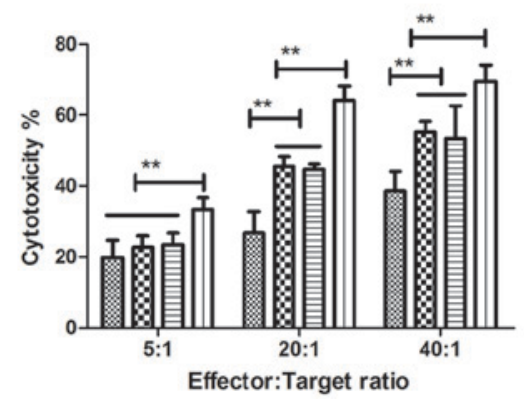

D

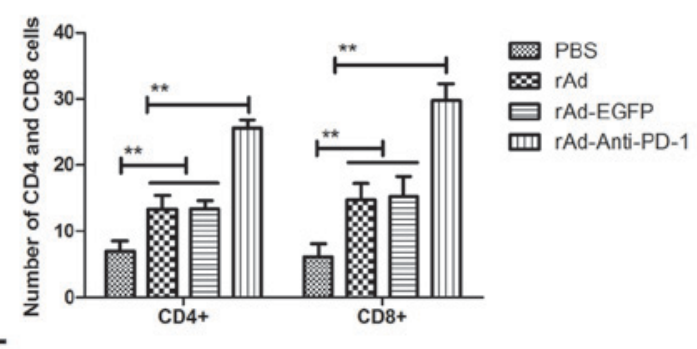

$\mathbf{F}$

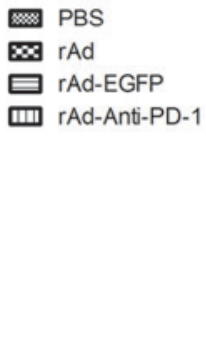

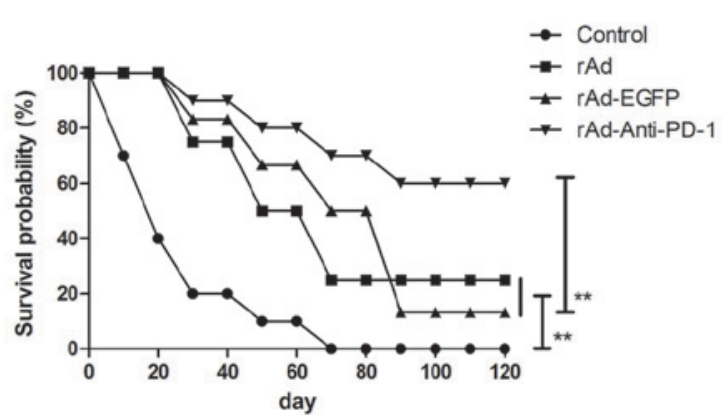

Figure 4. rAd-anti-PD-1 enhanced DCs to present melanoma tumor antigen. (A) Expression of tumor antigens and DC cells was assessed in tumors from experimental mice on day 30 (magnification, x40). (B) Expression of PD-1 and apoptotic bodies in tumors were analyzed after treatment with recombinant adenovirus (magnification, $\mathrm{x} 40$ ). (C) IFN levels were analyzed after treatment with recombinant adenovirus. (D) Level of CD4 ${ }^{+} \mathrm{CD} 8^{+} \mathrm{T}$ cells were assessed in tumors during treatment on day 30. (E) Analysis of the CTL responses in melanoma-bearing mice treated with the rAd-Anti-PD-1. (F) Tumor challenge experiment analyzed the long-term efficacy of rAd-anti-PD-1 treatment. Data are presented as mean \pm standard error of the mean. ${ }^{* *} \mathrm{P}<0.01$ vs. control. $\mathrm{rAd}$, recombinant adenovirus expressing; PD-1, programmed death-1; DC, dendritic cells; IFN, interferon; CD, cluster of differentiation; CTL, cytotoxic T lymphocyte; PBS, phosphate-buffered saline; EGFR, enhanced green fluorescent protein.

immunotherapy and induction of the cytotoxic T-cell immune responses in the majority of tumor cells (60). The data from the present study has identified that $\mathrm{Ad}$-Anti-PD-1 recognized PD-1 in tumor cells of mice with melanoma and decreased the expression of PD-1 and enhanced the infiltration of T cells. Although the induction of tumor cell apoptosis due to PD-1 inhibition or antibodies in patients with metastatic melanoma has been investigated, the PD-1 signaling pathway remains to be elucidated. Notably, the present study revealed that anti-PD-1, delivered by rAd-anti-PD-1, induces DC maturation and presents more melanoma-specific antigens for antitumor responses, which contribute to improved long-term survival. This may potentially lead to marked antitumor responses through activation of the immune system.

In conclusion, the present study demonstrates that full-length Anti-PD-1 may be expressed by rAd-Anti-PD-1-infected 
tumor cells and inhibits the growth and invasion of melanoma cells. In addition, the most notable finding in the present study is that anti-PD-1 expressed by rAd-anti-PD-1-infected tumor cells induces the maturation of DCs, which to the best of our knowledge has not been reported in previous studies. The rAd-anti-PD-1 virus is associated with a number of antitumor capacities and largely inhibits tumor growth in vivo, indicating its potential antitumor effects. Furthermore, rAd-anti-PD-1 induced the DC-presenting tumor antigen and promoted a more potent CTL in the immune response. Taken together, the use of DC-presenting tumor antigens as a strategy of rAd-Anti-PD-1 may represent a potential strategy of combining the oncolytic efficacy of adenovirus and present tumor antigen in addition to CTL, which enhance the anti-melanoma potency.

\section{References}

1. Ungureanu L, Botar Jid C, Candrea E, Cosgarea R and Senila SC: The role of lymph node ultrasound evaluation in melanoma-review of the literature. Med Ultrasono 18: 224-230, 2016.

2. Liu H, Yan Y and Jiang CM: Primary malignant melanoma of the esophagus with unusual endoscopic findings: A case report and literature review. Medicine (Baltimore) 95: e3479, 2016.

3. Linertová R, Valcárcel-Nazco C and Lacalle-Remigio JR: Management of benign melanocytic lesions as a melanoma prevention. Systematic review. Med Clin (Barc) 147: 162-170, 2016 (In Spanish).

4. Cazes A and Ronai ZA: Metabolism in melanoma metastasis. Pigment Cell Melanoma Res 29: 118-119, 2016.

5. Martínez-Esteve A, Álvarez-Pérez RM, Caballero-Gullón L, Sancho-Márquez MP and Borrego-Dorado I: Breast metastasis from melanoma mimicking inflammatory breast cancer. Eur J Nuc Med Mol Imaging 43: 389-390, 2016.

6. Margolin KA: Brain metastases in melanoma: Moving toward curing the incurable. J Oncol Pract 12: 545-546, 2016.

7. Chukwueke U, Batchelor T and Brastianos P: Management of brain metastases in patients with Melanoma. J Oncol Pract 12: 536-542, 2016

8. SchwartzH, BlacherE, Amer M,Livneh N, AbramovitzL, Klein A, Ben-Shushan D, Soffer S, Blazquez R, Barrantes-Freer A, et al: Incipient melanoma brain metastases instigate astrogliosis and neuroinflammation. Cancer Res 76: 4359-4371, 2016.

9. Sharma G, Lian CG, Lin WM, Amin-Mansour A, Jané-Valbuena J, Garraway L, Bao W, Yoon CH and Ibrahim N: Distinct genetic profiles of extracranial and intracranial acral melanoma metastases. J Cutan Pathol 43: 884-891, 2016.

10. Luo M and Fu L: The effect of chemotherapy on programmed cell death 1/programmed cell death 1 ligand axis: Some chemotherapeutical drugs may finally work through immune response. Oncotarget 7: 29794-29803,2016.

11. Fang XN and Fu LW: Predictive efficacy biomarkers of programmed cell death 1/programmed cell death 1 ligand blockade therapy. Recent Pat Anticancer Drug Discov 11: 141-151, 2016.

12. Dulos J, Carven GJ, van Boxtel SJ, Evers S, Driessen-Engels LJ, Hobo W, Gorecka MA, de Haan AF, Mulders P, Punt CJ, et al: PD-1 blockade augments Th1 and Th17 and suppresses Th2 responses in peripheral blood from patients with prostate and advanced melanoma cancer. J Immunother 35: 169-178, 2012.

13. Sakthivel P, Gereke M and Bruder D: Therapeutic intervention in cancer and chronic viral infections: Antibody mediated manipulation of PD-1/PD-L1 interaction. Rev Recent Clin Trials 7: 10-23, 2012.

14. Lee JJ, Chan A and Tang T: Tuberculosis reactivation in a patient receiving anti-programmed death-1 (PD-1) inhibitor for relapsed Hodgkin's lymphoma. Acta Oncol 55: 519-520, 2016.

15. Karakatsanis S,Bertsias G,Roussou PandBoumpas D:Programmed death 1 and $\mathrm{B}$ and $\mathrm{T}$ lymphocyte attenuator immunoreceptors and their association with malignant T-lymphoproliferative disorders: Brief review. Hematol Oncol 32: 113-119, 2014.

16. Huang B, Chen L, Bao C, Sun C, Li J, Wang L and Zhang X: The expression status and prognostic significance of programmed cell death 1 ligand 1 in gastrointestinal tract cancer: A systematic review and meta-analysis. Onco Targets Ther 8: 2617-2625, 2015.
17. Ferris R: PD-1 targeting in cancer immunotherapy. Cancer 119: E1-E3, 2013.

18. Yan F, Zheng Y and Huang L: Adenovirus-mediated combined anti-angiogenic and pro-apoptotic gene therapy enhances antitumor efficacy in hepatocellular carcinoma. Oncol Lett 5: 348-354, 2013.

19. Sinkovics JG and Horvath JC: Natural and genetically engineered viral agents for oncolysis and gene therapy of human cancers. Arch Immunol Ther Exp (Warsz) 56 (Suppl 1): S3-S59, 2008.

20. Zhang MM, Yan LN, Li DH, Gou XH, Liu JW, Su Z, Han L and Zhao LY: Inhibition of adenovirus-mediated gene transfer of antisense matrix metalloproteinase- 2 on hepatocellular carcinoma growth in vivo. Zhonghua Gan Zang Bing Za Zhi 13: 671-674, 2005 (In Chinese)

21. Thomas AA, Ernstoff MS and Fadul CE: Immunotherapy for the treatment of glioblastoma. Cancer J 18: 59-68, 2012.

22. Larsen CJ: Cellular immunotherapy and glioblastoma: A hopeful treatment? Bull Cancer 98: 457, 2011 (In French).

23. Varghese S, Rabkin SD, Nielsen GP, MacGarvey U, Liu R and Martuza RL: Systemic therapy of spontaneous prostate cancer in transgenic mice with oncolytic herpes simplex viruses. Cancer Res 67: 9371-9379, 2007.

24. Husain SR, Behari N, Kreitman RJ, Pastan I and Puri RK: Complete regression of established human glioblastoma tumor xenograft by interleukin-4 toxin therapy. Cancer Res 58: 3649-3653, 1998

25. Debinski W, Gibo DM, Obiri NI, Kealiher A and Puri RK: Novel anti-brain tumor cytotoxins specific for cancer cells. Nat Biotechnol 16: 449-453, 1998.

26. Bera TK, Viner J, Brinkmann E and Pastan I: Pharmacokinetics and antitumor activity of a bivalent disulfide-stabilized Fy immunotoxin with improved antigen binding to erbB2. Cancer Res 59: 4018-4022, 1999.

27. Ghetie MA, Richardson J, Tucker T, Jones D, Uhr JW and Vitetta ES: Antitumor activity of Fab' and IgG-anti-CD22 immunotoxins in disseminated human $\mathrm{B}$ lymphoma grown in mice with severe combined immunodeficiency disease: Effect on tumor cells in extranodal sites. Cancer Res 51: 5876-5880, 1991.

28. Costa Neves M, Giakoustidis A, Stamp G, Gaya A and Mudan S: Extended survival after complete pathological response in metastatic pancreatic ductal adenocarcinoma following induction chemotherapy, chemoradiotherapy, and a novel immunotherapy agent, IMM-101. Cureus 7: e435, 2015.

29. Li J, Chen QY, He J, Li ZL, Tang XF, Chen SP, Xie CM, Li YQ, Huang LX, Ye SB, et al: Phase I trial of adoptively transferred tumor-infiltrating lymphocyte immunotherapy following concurrent chemoradiotherapy in patients with locoregionally advanced nasopharyngeal carcinoma. Oncoimmunology 4: e976507, 2015.

30. Wang X, Bayer ME, Chen X, Fredrickson C, Cornforth AN, Liang G, Cannon J, He J, Fu Q, Liu J, et al: Phase I trial of active specific immunotherapy with autologous dendritic cells pulsed with autologous irradiated tumor stem cells in hepatitis B-positive patients with hepatocellular carcinoma. J Surg Oncol 111: 862-867, 2015

31. Nazarkina ZhK and Laktionov PP: Preparation of dendritic cells for cancer immunotherapy. Biomed Khim 61: 30-40, 2015 (In Russian).

32. Dias JD, Hemminki O, Diaconu I, Hirvinen M, Bonetti A, Guse K, Escutenaire S, Kanerva A, Pesonen S, Löskog A, et al: Targeted cancer immunotherapy with oncolytic adenovirus coding for a fully human monoclonal antibody specific for CTLA-4. Gene Ther 19: 988-998, 2012.

33. LaRocca CJ, Han J, Gavrikova T, Armstrong L, Oliveira AR, Shanley R, Vickers SM, Yamamoto $M$ and Davydova J: Oncolytic adenovirus expressing interferon alpha in a syngeneic Syrian hamster model for the treatment of pancreatic cancer. Surgery 157: 888-898, 2015.

34. Gerhardt A, Usener D, Keese M, Sturm J, Schadendorf D and Eichmuller S: Tissue expression and sero-reactivity of tumor-specific antigens in colorectal cancer. Cancer Lett 208: 197-206, 2004

35. Santin AD, Bellone S, Palmieri M, Bossini B, Cane' S, Bignotti E, Roman JJ, Cannon MJ and Pecorelli S: Restoration of tumor specific human leukocyte antigens class I-restricted cytotoxicity by dendritic cell stimulation of tumor infiltrating lymphocytes in patients with advanced ovarian cancer. Int J Gynecol Cancer 14: 64-75, 2004. 
36. Morotomi N, Fukuda K, Nakano M, Ichihara S, Oono T, Yamazaki T, Kobayashi N, Suzuki T, Tanaka Y and Taniguchi H: Evaluation of intestinal microbiotas of healthy Japanese adults and effect of antibiotics using the 16S ribosomal RNA gene based clone library method. Biol Pharm Bull 34: 1011-1020, 2011.

37. Gustafsson RK, Engdahl EE and Fogdell-Hahn A: Development and validation of a Q-PCR based TCID50 method for human herpesvirus 6. Virol J 9: 311, 2012

38. Livak KJ and Schmittgen TD: Analysis of relative gene expression data using real-time quantitative PCR and the 2(-Delta Delta C(T)) method. Methods 25: 402-408, 2001.

39. Fukui A, Muragaki Y, Saito T, Maruyama T, Nitta M, Ikuta S and Kawamata T: Volumetric analysis using low-field intraoperative magnetic resonance imaging for 168 newly diagnosed supratentorial glioblastomas: Effects of extent of resection and residual tumor volume on survival and recurrence. World Neurosurg 98: 73-80, 2017.

40. The Guide for the Care and Use of Laboratory Animals. ILAR J 57: NP, 2016.40. Greaves MF and Brown G: Purification of human T and B lymphocytes. J Immunol 112: 420-423, 1974

41. Shiono H and Ito Y: Novel method for continuous cell separation by density gradient centrifugation: Evaluation of a miniature separation column. Prep Biochem Biotechnol 33: 87-100, 2003.

42. Ting WH, Chien MN, Lo FS, Wang CH, Huang CY, Lin CL, Lin WS, Chang TY, Yang HW, Chen WF, et al: Association of cytotoxic t-lymphocyte-associated protein 4 (CTLA4) gene polymorphisms with autoimmune thyroid disease in children and adults: Case-control study. PloS One 11: e0154394, 2016.

43. Zamarin D, Vigil A, Kelly K, Garcia-Sastre A and Fong Y: Genetically engineered Newcastle disease virus for malignant melanoma therapy. Gene Ther 16: 796-804, 2009.

44. Allegra M, Zaragkoulias A, Vorgia E, Ioannou M, Litos G, Beug H and Mavrothalassitis G: Semaphorin-7a reverses the ERF-induced inhibition of EMT in Ras-dependent mouse mammary epithelial cells. Mol Biol Cell 23: 3873-3881, 2012.

45. Youlin K, Jian K, Siming L, Li Z, Weiyang H, Chaodong L and Xin G: Potent anti-prostate cancer immune response induced by dendritic cells transduced with recombinant adenoviruses encoding 4-1BBL combined with cytokine-induced killer cells. Immunotherapy 7: 13-20, 2015.

46. Leisegang M, Kammertoens T, Uckert $\mathrm{W}$ and Blankenstein $\mathrm{T}$ : Targeting human melanoma neoantigens by $\mathrm{T}$ cell receptor gene therapy. J Clin Invest 126: 854-858, 2016.

47. Braybrooke JP, Slade A, Deplanque G, Harrop R, Madhusudan S, Forster MD, Gibson R, Makris A, Talbot DC, Steiner J, et al: Phase I study of MetXia-P450 gene therapy and oral cyclophosphamide for patients with advanced breast cancer or melanoma. Clin Cancer Res 11: 1512-1520, 2005.
48. Zou W, Luo C, Zhang Z, Liu J, Gu J, Pei Z, Qian C and Liu X: A novel oncolytic adenovirus targeting to telomerase activity in tumor cells with potent. Oncogene 23: 457-464, 2004.

49. Hernández-Alcoceba R, Sangro B and Prieto J: Gene therapy of liver cancer. Ann Hepatol 6: 5-14, 2007.

50. Moss JA: Gene therapy review. Radiol Technol 86: 155-180; quiz 181-184, 2014.

51. Eibel B, Markoski MM, Rodrigues CG, Dipp T, de Salles FB, Giusti II, Nardi NB, Plentz RD and Kalil RA: VEGF gene therapy cooperatively recruits molecules from the immune system and stimulates cell homing and angiogenesis in refractory angina. Cytokine 91: 44-50, 2017.

52. Sharma A, Easow Mathew M, Sriganesh V and Reiss UM: Gene therapy for haemophilia. Cochrane Database Syst Rev 12: CD010822, 2016.

53. Hammer A and Steiner S: Gene therapy for therapeutic angiogenesis in peripheral arterial disease-a systematic review and meta-analysis of randomized, controlled trials. VASA 42 : 331-339, 2013.

54. Kumar MS, Masthan KM, Babu NA and Dash KC: Gene therapy in oral cancer: A review. J Clin Diagn Res 7: 1261-1263, 2013.

55. Koirala A, Conley SM and Naash MI: A review of therapeutic prospects of non-viral gene therapy in the retinal pigment epithelium. Biomaterials 34: 7158-7167, 2013.

56. Kim YS, Hwang KA, Go RE, Kim CW and Choi KC: Gene therapy strategies using engineered stem cells for treating gynecologic and breast cancer patients (Review). Oncol Rep 33: 2107-2112, 2015.

57. Watanabe M, Nasu Y and Kumon H: Adenovirus-mediated REIC/Dkk-3 gene therapy: Development of an autologous cancer vaccination therapy (Review). Oncol Lett 7: 595-601, 2014.

58. Ludlow SP, Andrews S, Pasikhova Y and Hill E: New-onset toxicity with programmed death-1 inhibitor rechallenge. Melanoma Res 26: 316-318, 2016.

59. Ansell SM: Where do programmed death-1 inhibitors fit in the management of malignant lymphoma? J Oncol Pract 12: 101-106, 2016.

60. Villasboas JC and Ansell S: Checkpoint inhibition: Programmed cell death 1 and programmed cell death 1 ligand inhibitors in Hodgkin lymphoma. Cancer J 22: 17-22, 2016. 